\title{
Reflexión sobre la memoria y autorreflexión de la historia
}

Hernán Sorgentini

Centro de Investigaciones Socio-Históricas

Universidad Nacional de La Plata

\section{RESUMEN}

Este artículo analiza la vinculación entre la reflexión sobre la memoria y diversas cuestiones que atañen a los aspectos epistemológicos, normativos y prácticos del conocimiento histórico. Considera específicamente la recepción de la obra de Maurice Halbwachs sobre la memoria colectiva en la autorreflexión de la historia trazada por Marc Bloch. Piensa también el papel de la memoria como objeto y función práctica de la historia y la definición de la historia como crítica de la tradición. Trabaja diferentes inflexiones de la relación entre memoria, historia y tradición en dos autores, E. P. Thompson y Carlo Ginzburg, cuyas obras resultan encuadrables en un registro de autorreflexión similar. Palabras clave: Historiografía, memoria, tradición.

\begin{abstract}
This article analyzes the link between the reflection on memory and many issues concerning the epistemological, normative and practical aspects of historical knowledge. It specifically considers the reception of Maurice Halbwachs's work related to the collective memory in the auto reflection of history drawn by Marc Bloch. Also it takes into account the role of memory as an object and an useful purpose of history and its definition as a criticism of tradition. It deals with different analysis of the association among memory, history and tradition of two authors, E.P. Thompson ad Carlo Ginzburg, whose works are placed in a similar entry of auto reflection.
\end{abstract}

Keywords: Historiography; memory; tradition.

Dentro de los vastos problemas que se sitúan en el marco de la tensión entre memoria e historia, este trabajo se propone revisar la vinculación entre la reflexión sobre la memoria y diversas cuestiones que atañen a los aspectos epistemológicos, normativos y prácticos del conocimiento histórico. Para ello, focaliza su atención en una línea de planteamientos programáticos y desarrollos historiográficos producidos en el marco de diversos debates e intervenciones registrados en el proceso de renovación de la disciplina histórica en el siglo XX y susceptibles de ser leídos en clave autorreflexiva, con el obje- 
to de aportar elementos para una conceptualización del oficio de historiador en un sentido no reducido a la observancia de una serie de pautas y procedimientos de carácter formal: más allá de la centralidad de éstos, el examen propuesto postula la posibilidad de considerar los aspectos ético-políticos intrínsecos a la práctica de reconstrucción del pasado, elucidar algunas de las formas de sus múltiples entrecruzamientos con el imperativo de comprensión y poner en discusión la pertinencia de la perspectiva de una crítica interna a la disciplina para establecer la potencialidad teórica de la historiografía en un sentido crítico de diversas concepciones que reducen sus aportes a la posibilidad de corroborar, matizar y/o proveer ejemplos a construcciones sistemáticas cuya formulación resulta siempre externa al registro de la propia doxa. De este modo, la vinculación entre reflexión sobre la memoria y autorreflexión de la historia se presenta como un espacio propicio para delinear herramientas analíticas adecuadas para enfrentar una serie de cuestiones centrales del debate actual sobre la conformación y transmisión de la(s) memoria(s) colectiva(s), entre las que pueden citarse, a título de ejemplos, la trama de continuidades y cambios que conforman la(s) tradición(es), los procesos de conformación y transmisión de "recuerdos falsos", las dificultades cognoscitivas de aproximación, registro y representación de experiencias vividas y recordadas como radicalmente "traumáticas" por los sujetos involucrados en ellas, las posibilidades y límites del conocimiento sistemático para dar cuenta de la perspectiva de las víctimas, la dimensión ética intrínseca al problema de la reconstrucción histórica y su vinculación con la función normativa de la noción de verdad, etc.

Significativamente, dentro de los múltiples estudios encarados en el contexto del creciente interés que está teniendo el tema de la memoria, aquellos trabajos encuadrados en el registro de la investigación histórica ocupan un lugar central, sobre todo como resultado de la recepción de distintas aproximaciones historiográficas que toman a las representaciones colectivas como un objeto privilegiado de la disciplina y, consecuentemente, habilitan la construcción de una historia de la(s) memoria(s); sin embargo, al momento de definir los problemas teóricos que encuadran estas investigaciones, la dimensión teórica implícita en los principales desarrollos programáticos sobre el conocimiento histórico y en la lectura de la historiografía no han sido opciones privilegiadas. En este sentido, nos proponemos rescatar la introducción de la cuestión de la memoria en el pensamiento historiográfico a partir de la obra de Marc Bloch - y su recepción crítica de la obra del sociólogo durkheimniano Maurice Halbwachs-, estableciendo su papel como objeto y función práctica de la historia y sus implicancias en la redefinición de la disciplina en un sentido crítico de la tradición, para luego dar lugar a un repaso de dos autores posteriores, E. P. Thompson y Carlo Ginzburg, cuyos desarrollos 
en el marco de nuevas aperturas teóricas, al poner en juego distintos aspectos de las relaciones entre memoria, historia y tradición, se tornan susceptibles de ser leídos, fuera de toda pretensión de reconstruir una genealogía, como tematizaciones de cuestiones afines en un registro de reflexión similar.

La elección de Marc Bloch como primer autor a considerar en este rastreo obedece antes que a su (debatible) carácter fundante de una nueva forma de hacer y pensar la historia a partir de la primera generación de la Escuela de los Annales, al registro específico desde el que este autor construye una "apología para la historia", es decir, al replanteamiento de las funciones de saber y memoria del conocimiento histórico particularmente visibles en su distinción de los problemas de la legitimidad y la utilidad de la historia, y a algunos de los contenidos de su historiografía articulados en torno a las cuestiones de la transmisión de la memoria y la crítica de la tradición. En este registro, a partir de la recepción de los planteamientos clásicos sobre la «memoria colectiva» del sociólogo durkheimniano Maurice Halbwachs, la memoria como objeto de la historia ocupa un lugar central, aportando elementos para un "examen de conciencia" de la disciplina que busca dar respuesta al tipo de críticas provenientes de distintas vertientes de pensamiento desde fines del siglo XIX ${ }^{1}$.

En su principal obra sobre el tema, Les cadres Sociaux de la Mémoire, Halbwachs opera un pasaje del tratamiento filosófico realizado por Henri Bergson sobre el problema de la memoria en términos de psicología individual a una consideración que, sin desentenderse del carácter filosófico del problema, lo reescribe en términos de las ciencias sociales, poniendo en el centro del análisis la "determinación social" y construyendo su objeto en torno a la(s) memoria(s) colectiva(s) de los grupos, de los que estudia específicamente la familia, la religión y la clase. Si Bergson concibe a la memoria en el horizonte de la duración como función de la conciencia individual en el espacio entre acción y representación, Halbwachs incorpora la lógica de los grupos en el contexto de un tratamiento en el que las representaciones colectivas - que condicionan y posibilitan la actividad de la memoria — vinculan las necesidades prácticas de estos grupos con la resistencia inercial de la tradición. De este modo, la memoria, circunscripta a una duración corta limitada a las generaciones vivas, se remonta a través del pensamiento hasta un cierto límite, concentrándose en las realidades idénticas a sí mismas y percibiendo su duración como continuidad entre representación y acción, pero sin resultar proclive a percibir los cambios que ocurren en la duración ${ }^{2}$. 
La reseña de Les cadres... que realiza Bloch en la Revue de synthèese encuentra en Halbwachs un pensamiento estimulante para el abordaje de temas hasta entonces poco explorados por la disciplina histórica, concentrándose en la importancia de centrar el objeto en fenómenos de carácter colectivo que serán característicos de desarrollos posteriores de la historiografía. Sin embargo, esta recepción, signada por el estímulo que el autor reconoce en la perspectiva sociológica, no impide a Bloch la puntualización de una serie de críticas a ésta, atentas a distinguir los puntos en que, con respecto a ella, diverge la perspectiva de la disciplina propia: Bloch acuerda con Halbwachs en la utilización de categorías de origen social, situadas en espacio y tiempo, pero no deja de reprochar el carácter limitado de una perspectiva centrada en un estudio de la memoria de los grupos, al que propone incorporar un análisis de la memoria jurídica y el derecho consuetudinario. Asimismo, establece una precisión con respecto a lo que entiende como una transpolación de conceptos propios de problemas de psicología individual a problemas del análisis social. Ambos elementos convergen en una perspectiva histórica del problema que reclama un enfoque centrado en los mecanismos de transmisión de la memoria a partir del reconocimiento de que "una parte de los fenómenos que así designamos [como memoria colectiva] son simplemente cuestiones que tienen que ver con la comunicación entre los individuos", aportando elementos para percibir los errores en la transmisión de los recuerdos y para registrar los cambios que ocurren dentro de las tradiciones bajo la apariencia de una continuidad ${ }^{3}$.

Las críticas de Bloch apuntan contra la concepción todavía tradicional que el propio Halbwachs mantiene sobre la objetividad e imparcialidad del conocimiento histórico. Incluso en un texto posterior a la creación de la revista Annales - de la que Halbwachs también participa - el examen de la conexión entre memoria e historia realizado por el sociólogo durkheimniano se construye sobre una oposición marcada entre memoria colectiva (interesada en el presente, multiforme y portadora de un sentido de identidad) e historia (interesada en el pasado, universal y portadora de un sentido de la diversidad). Más que en el carácter oposicional del vínculo y en la apelación al carácter objetivo de la historia, el problema de la consideración halbwachsiana reside en la tensión entre esta objetividad "ingenua" y la constatación de la existencia de una función práctica de la historia detectada a la luz de la exploración de las funciones de la memoria. Para Halbwachs, el registro de esta oposición no impide señalar que el objetivo de la historia es precisamente tender un puente entre el pasado y el presente, "reestablecer la continuidad interrumpida", a través del registro de "los acontecimientos dignos de memoria que constituyen la tradición y que exceden la restringida situación espacio temporal de los grupos que animan y mantienen viva la memoria social". La 
historia se define como la "memoria universal del género humano", resultando complementaria de la memoria de los grupos, en tanto "es el recuento de hechos que han ocupado el lugar más grande en la memoria de los hombres" y se justifica por la necesidad de fijar por escrito los recuerdos que no permanecen vivos para los grupos sociales ${ }^{4}$.

La historia como mecanismo de reestablecimiento de la continuidad de la tradición supone entonces dos cuestiones difíciles de admitir para la perspectiva del historiador: por un lado, que la tradición es continua; por otra parte, que la perspectiva universal de la historia no entraña una crítica a la tradición. Son precisamente estos puntos los que encontramos en los señalamientos de Bloch y en su tratamiento historiográfico. El enfoque centrado en los mecanismos de transmisión de la memoria reclamado por Bloch en su texto de 1925 entronca con una serie de reflexiones que el autor desplegó a lo largo de los años veinte en varios ensayos historiográficos. Estos ensayos exploran desde problemas indiscutiblemente "actuales" como la conformación y transmisión de noticias falsas durante la primera guerra mundial, hasta los sucesivos avatares en que se constituye y transforma la leyenda del rey Salomón en el espacio de la memoria, articulándose en torno al problema de la transmisión de los recuerdos e incorporando la cuestión de la verdad a la preocupación halbwachsiana sobre las "representaciones colectivas", a partir de la pregunta sobre cómo se generan y transmiten los "recuerdos falsos"

En una obra posterior, La sociedad feudal, Bloch plantea más específicamente la necesidad de un estudio histórico de la memoria colectiva, entendiéndola como un rasgo central para comprender la estructura social de las sociedades medievales. En un capítulo dedicado especialmente al tema, titulado "La memoria colectiva", el autor realiza una exploración de las imágenes del pasado presentes en las formas de la historiografía y la epopeya en el medioevo, a partir de la cual extrae una reflexión de proyecciones teóricas que establece una contraposición entre la actitud tradicionalista "que sin cesar atrae el presente hacia el pasado y con ello produce la confusión entre los colores de ambos" y "el espíritu histórico, dominado por el sentido de la diversidad". De esta distinción se infiere nuevamente que la perspectiva histórica es necesariamente crítica de la tradición, en tanto no puede perder de vista los errores en la transmisión de los recuerdos que ya el historiador había reprochado al sociólogo algo más de una década atrás.

Esta serie de trabajos constituyen una referencia central para comprender el sentido en que una reflexión crítica sobre las formas de transmisión de la memoria permean la reflexión sobre la historia trazada por el autor en su manuscrito incompleto elaborado en el contexto de la ocupación alemana, Apología para la historia o el oficio de historiador, texto que, a partir de una recepción que hoy se descubre como sesgada, ha marcado un hito en las nuevas 
formas de concebir la disciplina que empezarán a consolidarse tras la segunda guerra mundial. En este texto, la crítica de la memoria referida al problema de los actos de comunicación, asume un nuevo énfasis que se orienta a pensar este problema como central para la comprensión de las funciones de memoria de la disciplina histórica. La reflexión sobre la transmisión de la memoria se anuda con la pregunta por el sentido de la práctica historiográfica que recorre la Apología..., alcanzando su expresión más clara en la distinción de los problemas de la legitimidad y la utilidad de la historia, distinción en la que la función de memoria de la historia, pensada como un «acto de comunicación» desde la pregunta fundamental sobre la utilidad de la historia, se anuda con la función de saber de la disciplina, construida desde una redefinición de la legitimidad orientada por su vocación a la comprensión. El imperativo de "comprender y no juzgar", a partir del cual se define la legitimidad de la disciplina histórica, no implica un abandono de la pregunta sobre la utilidad de la historia, pero alerta sobre las consecuencias de la falacia de una resolución del problema de la función práctica del conocimiento histórico anteponiendo esta pregunta a aquella sobre las credenciales con que la disciplina debe poder contar para encararla efectivamente. La función de identidad de la historia no queda entonces excluida del planteamiento de Bloch, pero remite a un segundo momento que requiere la consideración previa de la historia misma. La historia ya no es "la memoria universal del género humano" como pretendía Halbwachs; en todo caso aspira a la consecución de una "memoria universalista" que expresa, antes que un encuentro con la tradición, la necesidad de un examen crítico de los mecanismos de su transmisión y configuración a través de los recuerdos colectivos ${ }^{7}$.

Esta divergencia entre la perspectiva histórica y la perspectiva sociológica remite, en último término, a una crítica al carácter arbitrario de la separación entre pasado y presente, es decir, a una consideración distinta del tiempo histórico. Para Bloch, es la distinción entre la memoria de las generaciones vivas (presente/pasado reciente) y el pasado muerto de la historia escrita (pasado lejano) la que todavía resulta arbitraria, ya que si el presente es "en lo infinito de la duración, un punto minúsculo que sin cesar se esquiva" y "en el lenguaje común, presente significa pasado reciente", la distinción entre pasado lejano y pasado reciente no deja de resultar problemática, en tanto "la noción de proximidad no sólo carece de precisión [...] sino que también nos coloca en presencia del más efímero de los atributos" ${ }^{8}$. Bloch remarca no sólo que el límite entre lo actual y lo inactual no puede establecerse a partir de una medida matemática del tiempo, sino que la necesidad práctica del presente compromete al conocimiento histórico en su conjunto. El pasado es algo igualmente vivo que el presente, porque la representación que la historia hace de él no se comprende fuera de un sentido que es el sentido del presente. Para la 
historia, el tiempo no puede ser pensado sino como "parte constitutiva de[1] objeto", o más precisamente como su "medio y la materia concreta", en tanto no sólo tiende a conformar su objeto, sino que además es el presupuesto de las posibilidades del análisis por medio del cual el historiador establece un ordenamiento racional del pasado: "el plasma mismo donde están sumergidos los fenómenos y [...] el lugar de su inteligibilidad". Y el lenguaje por medio del cual el historiador construye su conocimiento sobre el pasado no puede ser pensado fuera de su existencia temporal: es el medio esencial para el objetivo de la comprensión, en tanto a través de él el historiador realiza los procedimientos de selección, ordenamiento racional y análisis de los hechos históricos, y en tanto constituye la materia de los testimonios a través de los cuales el historiador accede al pasado. Si en el contexto de su reformulación del conocimiento histórico Bloch considera provechosa la remisión sociológica a las distintas existencias espacio-temporales de las formas de memoria de los grupos, lo hace desde el supuesto que el tiempo materia no sólo excede la indistinción entre pasado y presente, sino que además constituye el supuesto del ordenamiento racional del pasado operado a través de las categorías históricas?.

La concepción diferente del tiempo histórico que entraña la idea de "plasma" redefine entonces la relación entre la tradición y el objetivo universalista de la historia. En Halbwachs el universalismo de la historia resulta abstracto ya que, al no implicar una crítica al particularismo de las identidades de los grupos, termina tornando problemática la expresión "memoria histórica", por no poder conciliar la objetividad del conocimiento con su función práctica. En el caso de Bloch, el intento de concebir una "memoria universalista" refiere a una idea de humanidad que se asienta sobre una perspectiva crítica de la tradición y en una concepción distinta del tiempo en la que el tiempo concreto y real es la materia misma de la historia.

Otro texto, contemporáneo de la Apología..., permite establecer con más claridad las implicancias de este universalismo redefinido. En L'étrange défaite es posible observar cómo el universalismo de Bloch no implica un abandono de la fidelidad a la tradición nacional, en cuyos horizontes el autor redefine - en sus textos programáticos — una ciencia que se propone como fin último contribuir a que los hombres vivan mejor. Sin embargo, el vínculo entre esta tradición y la perspectiva del historiador se sitúa en un lugar muy preciso, justamente en aquel punto en que aquella tradición nacional tiene de particular, su real vocación universalista: Bloch es, al fin de cuentas, un historiador que piensa la historia desde la historia misma, en la tradición de la revolución francesa. La función práctica de la historia como memoria colectiva remite a una comunidad que le permite identificarse cómodamente como un 
"ciudadano francés" y en la que sólo en un caso reivindica su condición de judío: frente a un antisemita ${ }^{10}$.

La introducción del problema de la memoria en el pensamiento historiográfico y sus implicancias en el plano de una autorreflexión de la historia construida a partir de una perspectiva disciplinar atenta a los mecanismos de transmisión de la memoria y a los aspectos cambiantes de la tradición, rastreadas en la obra de Bloch, guiarán la exploración del segundo de los autores seleccionados, el historiador marxista británico E. P. Thompson. Si en Bloch la crítica a la concepción tradicional de la función de memoria de la historia se orientaba a redefinir esta función articulándola con la función de saber de la disciplina, en Thompson la introducción del sesgo recuperativo de la(s) experiencia(s) en torno al problema de la subjetividad en la historia genera una nueva inflexión, redefiniendo el problema de la(s) tradición(es) y extrayendo nuevas consecuencias con respecto a la función práctica de la historia, en este caso, desde desarrollos inscriptos en el marco de la exploración de las tensiones entre 'tradición y conocimiento científico/crítico', 'historiografía y teoría', 'valoración y objetividad', etc., resueltas de diversas maneras tanto en las versiones tradicionales de la disciplina como en aportes teóricos provenientes alternativamente de los universos de las ciencias sociales y las distintas versiones del marxismo, perspectivas todas con las que el autor discute.

Ha sido ya suficientemente señalada la multiplicidad de sentidos a que alude el concepto thompsoniano de «experiencia», básicamente en su doble carácter, determinado por el ser social y determinante de las formas de la conciencia social. Este doble carácter, impugnado alternativamente a partir de posiciones que subrayan cada uno de sus aspectos, no resulta contradictorio si se atiende al contexto de su formulación teórica en el marco de una reflexión construida desde la propia práctica historiográfica, en la que los componentes recuperativos del pasado, presentes en ambos sentidos de la «experiencia», se conectan - a través del reconocimiento del papel activo y la racionalidad de los sujetos que hicieron la historia y la propia valoración del historiador como elementos intrínsecos al proceso de reconstrucción histórica - con una discusión de la teoría marxista y de los criterios normativos del oficio de historiador ${ }^{11}$.

Thompson apunta a construir desde el conocimiento histórico un tipo de crítica que se desarrolla en el espacio de la tensión entre, por un lado, la recuperación moral y política de las razones, sentimientos y aspiraciones de los sujetos oprimidos en el pasado que estudia y el consecuente riesgo de una 
potencial identificación transhistórica con las formas de su racionalidad y, por otra parte, el imperativo de un pensamiento anclado en la tradición crítica de la razón y sus potenciales aspectos clausurantes. En la línea de un tipo de crítica que enfatiza la recuperación de lost rights, prefigurada por una generación anterior de marxistas británicos ${ }^{12}$, el sesgo recuperativo y crítico del concepto de «experiencia» apunta a señalar el papel activo de los sujetos como hacedores de la historia, validándose en su capacidad de hacer inteligibles aspectos oscuros del pasado desatendidos por las concepciones dominantes, como el sentido de la economía «moral» de los pobres en el siglo XVIII, el impacto a largo plazo de la tradición jacobina inglesa, la racionalidad de las prácticas luditas y el consecuente reconocimiento de su entidad política, etc. Por otra parte, la consideración de estos aspectos oscuros del pasado se concibe desde una perspectiva articulada en torno a los propios procedimientos de la reconstrucción histórica que, desde el reconocimiento del carácter proyectivo de la propia «experiencia» del historiador, se orienta a replantear la imagen general del pasado trazada por las interpretaciones que definen el oficio de historiador desde el supuesto de una objetividad a-valorativa. La focalización thompsoniana en la «experiencia» habilita de este modo una reconsideración de la(s) tradición(es) como sustrato de la constitución de la subjetividad en el contexto de una formulación en la que la intención reconstructiva del historiador supone una opción a la vez epistemológica y ético-política capaz de resituar los términos de la oposición trazada por Bloch entre "tradición» y «espíritu histórico» y, en un sentido más general, de reformular la antítesis entre juicio y comprensión sobre la que este autor definía la legitimidad de la práctica historiográfica.

Para dar cuenta de estas dos cuestiones tomaremos algunos de los núcleos más significativos de sus obras historiográficas. En La formación de la clase obrera en Inglaterra, Thompson concibe las tradiciones populares del siglo XVIII como un elemento clave para reconstruir el substrato sobre el que se conforma la clase como sujeto, en tanto estas tradiciones constituyen la materia prima a partir de la cual opera la "determinación" de las relaciones de producción, a la vez que dan cuenta de un cúmulo de experiencias históricas que las nuevas formas de conciencia que definen el proceso de formación de la clase obrera - articuladas en las formas de autoorganización de "innumerables miembros" y en el cuestionamiento de los derechos de propiedad - incorporan y transforman radicalmente a partir de la ruptura política marcada por el jacobinismo inglés de la década de 1790. En este sentido, la recuperación de estas tradiciones opera como un correctivo contra la importación de un modelo clásico de conciencia de clase autovalidado teóricamente - tanto en sus versiones más deterministas como en aquellas de matriz hegeliana - inscribiéndose en una conceptualización histórica de la conforma- 
ción de la clase y cuestionando la identidad entre conciencia de clase y práctica revolucionaria; pero, a la vez, al tratarse de una recuperación selectiva en este caso las tradiciones recuperadas se circunscriben a la tradición de disidencia y sus modificaciones en el contexto del resurgimiento metodista, la tradición de la multitud del siglo XVIII y la tradición de nociones populares asociadas a la idea del «inglés libre por nacimiento» - mantiene la idea de una conciencia unitaria que valida el concepto (y la existencia) de la clase obre$\mathrm{ra}$ frente a los aspectos disolutivos de esta unidad provenientes de las ciencias sociales afines al concepto de "clases trabajadoras» y a las revisiones del economicismo marxista que rechazan el canon interpretativo «base/superestructura» en términos de una indeterminación: una tesis central del libro es aquella que postula que es la «experiencia de clase» la que confiere sentido a una serie de prácticas y valores de los hombres y mujeres que vivieron y sufrieron el doble proceso de explotación económica y opresión política a través del cual se constituyen como sujetos y que la recuperación de esta «experiencia de clase» permite al historiador cuestionar los juicios implícitos en las interpretaciones historiográficas de signo liberal y, con ello, ampliar el conocimiento del proceso en su conjunto ${ }^{13}$.

De esta tesis se desprende una consideración sobre el tipo de función de memoria que Thompson asigna a la historia: ésta busca rescatar a aquellos hombres y mujeres de "la enorme condescendencia de la posteridad" (como señala su más citada frase del Prefacio de La formación...), no como una suerte de resarcimiento póstumo para con las víctimas, sino como una tarea inexcusable del presente, ya que una interpretación que los mantenga en el olvido haría que sigan siendo víctimas y obliteraría, de este modo, las posibilidades de la clase obrera de reconocerse en ellas como sujeto activo de la historia.

La intelección del componente activo presente en la 'tradición de los oprimidos' sólo es posible desde una postura ética que respeta las razones, sentimientos y valores de aquellos hombres y mujeres, y que asume el carácter político que orienta su recuperación desde el presente. Así, por ejemplo, en el caso de la reconstrucción del fenómeno ludita, Thompson reconoce desde un punto de vista moral la validez de las acciones de quienes participaron del movimiento $y$, consecuentemente, desde el punto de vista político, combate las interpretaciones dominantes que asumen como un presupuesto la validez de los juicios implícitos de los reformadores contemporáneos que desestiman la racionalidad del fenómeno. Junto con la negación de esta racionalidad contraria a la imposición disruptora del laissez faire y alternativa a la que finalmente triunfó - estas interpretaciones desestiman la entidad histórica del fenómeno, extrapolando la lógica de razonamiento político de los sujetos que sostuvieron alternativas triunfantes en lógica de la razón histórica. En cambio, la opción autoconsciente del propio posicionamiento político que sostie- 
ne Thompson articula en dos instancias sucesivas la actitud de respeto moral frente a los sujetos que fueron protagonistas de la historia con el imperativo de comprensión de la práctica historiográfica: la crítica interna al partidismo de las fuentes en el registro de los procedimientos propios del oficio de historiador y, a partir de ella, la crítica interna a las interpretaciones liberales o reformistas que asignan un carácter necesario al proceso contingente por el cual la racionalidad del laissez faire, es decir, la racionalidad de algunos sujetos, resultó victoriosa. De este modo, descubre en la racionalidad moral de la oposición ludita al laissez faire una racionalidad política que constituye un antecedente de la "economía política de la clase obrera»: "Fue Marx quien vio, en la aprobación de la ley de las 10 horas (1847), una prueba de que "por primera vez ... en pleno día, la economía política de la clase media ha sucumbido a la economía política de la clase obrera». Los hombres que atacaron la fábrica de Cartwright en Rawfolds anunciaban esta economía política alternativa, aunque lo hiciesen en un confuso encuentro a medianoche"14.

Por otra parte, al igual que en el caso de Bloch, la función de memoria de la historia se conecta con un tratamiento específico de la memoria como objeto de la historia, en su caso, en el mismo registro de una crítica interna a los juicios implícitos sobre los que se construye la memoria social de opciones políticas que no resultaron victoriosas en el desarrollo del proceso histórico. La recuperación de la racionalidad alternativa de los luditas va asociada con el reconocimiento de la efectiva existencia de una "tradición clandestina" y de su entidad política negadas no sólo por las opciones reformistas contemporáneas, sino también por la memoria social que se constituye a partir de la derrota del movimiento. En este sentido, la inspección crítica de la memoria sesgada por el propio silencio de los protagonistas en las décadas siguientes a su derrota actúa como un elemento que, en la línea de los procedimientos de la crítica documental, reafirma la existencia de los hechos reconstruidos: "El ludismo acababa en el patíbulo; y en cualquier momento de los siguientes 40 años, declarar que uno había sido un instigador ludita podía atraer la atención, nada bienvenida, de las autoridades, y quizás incluso las recriminaciones de la comunidad en la que todavía vivían los familiares de aquellos que habían sido ejecutados. Los luditas que habían dejado atrás su pasado tenían tan pocas ganas de que les recordasen su juventud como un hombre que tenga antecedentes delictivos" 15 .

La conclusión de Thompson remite, antes que a dar cuenta de la memoria en el universo de las representaciones sociales, a lo que prueba la existencia de esta memoria, aún con sus rasgos deformadores y conscientemente autolimitados: esto es, a una experiencia histórica, la existencia de una tradición clandestina que, remontándose hacia atrás más allá de la represión de los años de las guerras napoleónicas hasta el jacobinismo, conecta a esta tradición con 
las luchas políticas de posguerra y, a través de ellas, con la consolidación de una conciencia de clase en el cartismo. En este sentido, la memoria importa no sólo en virtud de un análisis de los aspectos controversiales de un acontecimiento del pasado de complejas pervivencias en las décadas posteriores o de los mecanismos de afirmación de un determinado orden social en el presente a partir de la continua resignificación del pasado, sino más allá de ellos, como elemento que confirma la existencia de esos aspectos del proceso histórico que los contemporáneos tendieron a ocluir o subestimar y que resultan, por tanto, de difícil intelección para los historiadores. La apuesta por la reconstrucción histórica - limitada por la imposición de una imagen del pasado que el mismo desarrollo del proceso histórico tendió a entronizar - responde, de este modo, al imperativo de restituir un pasado para la conciencia de quienes forman parte, en el presente, de aquella 'tradición de los oprimidos'.

Puede observarse entonces un doble aspecto de la relación entre historia y memoria. Por un lado, la afirmación de la historia como crítica de los aspectos falaces de la memoria en la línea de la historia como crítica de la tradición propia de la perspectiva historiográfica; por otra parte, un aspecto iluminador de la memoria asociado con la valoración de los componentes positivos de las orientaciones potencialmente progresivas discernibles en las tradiciones que la «experiencia de clase» toma y a la vez resignifica, aspecto que aparece vinculado al reconocimiento de un punto de vista del historiador y a la legitimidad de emitir un juicio con respecto a los hechos del pasado.

La remisión posterior de la obra de Thompson al siglo XVIII puede leerse también en relación con el problema de la función de memoria de la historia, es decir, como un intento de iluminar el pasado oprimido de la clase obrera y rescatar una memoria de la resistencia y la lucha frente a la opresión con el fin de esclarecer nuevos sentidos en las formas de lucha a través de la cual se constituye la clase obrera en el período 1790-1832. La postulación de formas de "lucha de clases sin clases" en un polémico artículo de 1978 que rescata la noción de lucha de clases en un sentido amplio ${ }^{16}$ constituye una pieza central para comprender la ruptura ulterior dada por el proceso de conformación de una conciencia de clase definitoria del «ser» de la clase, en un sentido que registra los aspectos problemáticos de la tensión derivada de los postulados clásicos entre conciencia de clase como 'conjunto de intereses comunes' y como 'conciencia/práctica revolucionaria'. Por otra parte, abre un nuevo universo de debates en el que se sitúa la exploración de la cultura popular del siglo XVIII.

En el contexto de estos debates, Thompson se orienta a problematizar la cuestión de la «tradición» $y$, significativamente encuentra, al igual que Bloch, un referente empírico central en los aspectos cambiantes del derecho consuetudinario, referencia que le permite sostener sus reformulaciones teóricas. En 
Costumbres en común, la postulación de la existencia de una "conciencia de la costumbre" permite ver que "algunas "costumbres» eran inventos recientes y, en realidad, constituían la reivindicación de nuevos "derechos»". Este tratamiento busca corregir el sentido de "permanencia fija que sugiere la palabra «tradición»", considerando a la «costumbre» como un espacio de lucha, como "un campo de cambio y de contienda, una palestra en la que intereses opuestos hacían reclamaciones contrarias", y apunta a percibir a la costumbre en el marco una "cultura tradicional rebelde", de formas conservadoras y contenidos potencialmente transformadores ${ }^{17}$.

Las consideraciones ético-políticas que subyacen al posicionamiento de Thompson, orientadas a respetar la validez de ciertas prácticas de esta cultura tradicional rebelde resultan centrales para definir el marco de una exploración histórica fructífera de los aspectos cambiantes de la tradición, exploración sostenida a partir de una concepción de la cultura que diverge de la remisión reduccionista a la esfera de la «superestructura», y aún de expresiones teóricas en otros puntos convergentes de la nueva izquierda británica, como lo atestigua una polémica de vieja data con la concepción de cultura como "whole way of life" de Raymond Williams, recusada en términos netamente marxistas desde un concepto de cultura como "whole way of struggle". Por otra parte, los desarrollos de la historiografía thompsoniana permiten observar cómo en el marco de la apertura marcada por la concepción de una "cultura tradicional rebelde", la adscripción al marxismo actúa como una contención con respecto a una potencial identificación transhistórica con los valores del contenido rebelde de esta cultura. Aún cuando muchas de las tradiciones del siglo XVIII son recuperadas positivamente en razón de la resignificación que adquieren en el nuevo contexto de fuerzas a partir del cual se delimita el proceso de conformación de una conciencia de clase desde la ruptura política marcada por el jacobinismo, los parámetros del historiador permanecen en el universo de los estrechos marcos que establece esta nueva forma de conciencia sustentada en la «experiencia de clase» y no inhiben la emisión de un juicio basándose en una razón externa a la racionalidad de ciertas prácticas como la venta de esposas y la cencerrada ${ }^{18}$.

Así, si la perspectiva de Thompson habilita una reconsideración de la «tradición» como antítesis de la «conciencia» (o la perspectiva crítica de la historia), lo hace desde un registro teórico que reivindica explícitamente una lectura del marxismo como «tradición», una «tradición marxista» defendida en términos de un «materialismo histórico» fuertemente identificado con la perspectiva de la disciplina histórica y divergente, a partir de su constitución en este registro particular, de las múltiples formas de concebir al marxismo con las que disputa ${ }^{19}$. En esta concepción, la exploración del problema teórico de la subjetividad en la historia como problema del conocimiento históri- 
co articula un replanteamiento de los aspectos epistemológicos, normativos y prácticos del oficio de historiador: el objetivo de la comprensión no puede ya eludir la apelación al juicio y la función práctica de la historia no puede concebirse sino como función de un sujeto, inscripto en una tradición de vocación universalista, que está también en la historia.

\section{III}

La obra de Thompson es reconocida como referencia central del desarrollo de los estudios de la 'historia desde abajo', un heterogéneo abanico de perspectivas que procuran continuar el imperativo de memoria que implica rescatar a los hombres y mujeres que hicieron, vivieron y sufrieron la historia. En la recepción de su obra, el carácter recuperativo de la «experiencia» tiende a proyectarse, en principio, hacia una variedad de «experiencias» que contemplan otros sujetos no necesariamente inscriptos en la «experiencia de clase», como la 'gente corriente' y las mujeres; y, posteriormente, define una nueva agenda de temas que comprenden sucesivos vuelcos hacia los problemas de las identidades y las «subculturas», muchas veces en términos que universos discursivos, temas que se inscriben en el contexto de preocupaciones divergentes de la pregunta por el sujeto en clave thompsoniana y más bien responden a la multiplicación de sujetos hecha evidente a partir de las revueltas de $1968^{20}$.

La intención de constituir una memoria de 'los de abajo' a partir del rescate de una multiplicidad de experiencias desestimadas por las interpretaciones historiográficas dominantes afirma y amplía el postulado thompsoniano acerca de la necesidad de un reconocimiento del punto de vista del historiador y sus supuestos éticos con respecto a los sujetos que estudia; sin embargo, el encuadre de su recuperación desde el presente, ya no determinado por el sentido unitario de la «experiencia de clase», deja de privilegiar aquellas experiencias políticas y tiende a concentrarse en los modos de vida, la cotidianeidad, la cultura, etc. Esto no implica un abandono del sentido político que guía la intención recuperativa de estos estudios, pero éste tiende a desprenderse de su vinculación con la perspectiva universalista de la historia y las consecuencias que ésta tenía en relación con la autorreflexión sobre los aspectos normativos y prácticos del oficio ${ }^{21}$. En este sentido, aunque en muchos casos continuadores del proyecto de historiografía socialista de Thompson, estos planteamientos (sobre todo los desarrollados en el ámbito británico y posteriormente el mundo angloparlante), convergen más fácilmente con el sentido crítico contracultural de una práctica intelectual construida a partir de parámetros metadisciplinarios de algunos de los desarrollos de los nuevos 
estudios culturales - cuya concepción de cultura Thompson había rechazado explícitamente a partir de la reivindicación del concepto de «lucha de clases» en su polémica con Williams - o, simétricamente, al momento de establecer los horizontes teóricos que guían su práctica tienden a fundamentar su opción en producciones externas al registro de la disciplina ${ }^{22}$.

En el plano de la vinculación de la reflexión sobre la memoria con la autorreflexión sobre la historia, estos planteamientos pueden inscribirse en la tendencia a reconocer los aspectos de memoria de la práctica historiográfica en la línea de una reconsideración de la oposición tajante entre historia y tradición. Así, por ejemplo, en una obra dedicada al tema, Raphael Samuel define el tipo de conocimiento histórico que propone como un conocimiento "cuyos cursos son promiscuos, dibujados no sólo sobre la experiencia de la vida real, sino también sobre la memoria y el mito, la fantasía y el deseo; no sólo sobre el pasado cronológico de los recuerdos documentados, sino sobre el pasado sin tiempo (timeless) de la 'tradición"'23. Sin embargo, la articulación de su obra en torno a la cuestión de la subjetividad previene contra su inclusión dentro de la tendencia reductiva de la historia a un "lugar de memoria" más entre otros.

Esta tendencia reductiva parece ser, no obstante, el principal carril por el que discurre el reconocimiento de la dimensión de memoria de la historia en las tendencias contemporáneas a estos planteamientos. Como es sabido, el desarrollo y consolidación de la revista Annales no tendió a remarcar la vinculación del estudio de la memoria con la autorreflexión sobre la historia, pese a la centralidad que ésta tuvo en los señalamientos programáticos de Bloch. En general, los primeros trabajos que trazan una evolución sobre la corriente realizan una observación general sobre la vinculación entre Halbwachs y Bloch $y$, para referirse a la exploración del tema en la tradición francesa, remiten a Pierre Nora y su obra colectiva Les lieux de mémoire, anclada en el universo de preocupaciones de la tercera generación de la corriente.

En el Prólogo a la obra colectiva reunida bajo su dirección, Pierre Nora sitúa la exploración de los "lugares de memoria" como una preocupación que surge, por un lado, como resultado de un movimiento puramente historiográfico que convirtió a la historia en una ciencia social y remitió a la memoria a la esfera de lo privado y que ahora encuentra el momento de "un retorno reflexivo de la historia sobre sí misma" y, por otra parte, de un movimiento histórico en el que percibe el fin de "una tradición de memoria" como resultado de la "aceleración de la historia". Al presentarse a sí mismo como el testimonio del fin de una "tradición de memoria", esto es, "de la adecuación entre la historia y la memoria”, el planteamiento de Nora recepta en forma directa, más allá de la consideración de cualquier tratamiento historiográfico previo, la oposición entre memoria e historia trazada por Halbwachs; de esta mane- 
ra, su planteamiento alternativamente desconoce el registro particular en que la renovación historiográfica incorpora las aperturas teóricas provistas por las ciencias sociales y asocia toda concepción de la memoria como función práctica de la historia con las funciones de identidad y el sentido conmemorativo propios de la concepción tradicional de la disciplina mantenida por este autor ${ }^{24}$.

La perspectiva crítica propuesta por Nora cobra desarrollo entonces en el marco de diversas oposiciones: 'historia totémica-historia crítica', 'historia memoria-historia epistemológica', etc.. El concepto de los "lugares de memoria" - a partir del cual se conciben estas oposiciones - postula una ruptura con respecto a la continuidad implícita en una historia vinculada con la tradición nacional y se representa a sí mismo remitiendo la reflexión sobre la historia al umbral de la trascendencia de su función de identidad, en vistas a colocarla en su etapa "epistemológica": en su triple sentido, material, simbólico y funcional, anudado en última instancia en su posibilidad de ser reducido a un signo en estado puro, este autor concibe teóricamente a la historiografía existente como un lugar de memoria entre otros. De este modo, la reflexión sobre la memoria se inscribe en un lugar preciso de la curva de evolución de la disciplina volcada ahora a la indagación de las "representaciones colectivas" y, a partir de este giro, acarrea en el plano de la autorreflexión de la disciplina consecuencias claramente divergentes de las introducidas por Bloch a partir de la distinción de los problemas de la legitimidad y la utilidad de la historia, ya que, o bien los "lugares de memoria" son meramente objetos de la historia en el plano de su instrumentalización como concepto, o bien son instancia de trascendencia epistemológica de cualquier intención de su articulación como función práctica.

La obra del historiador italiano Carlo Ginzburg, el tercero de los autores a analizar, cobra relevancia en el contexto de, por un lado, la ampliación y pluralización del objeto de la historia desde abajo y su tendencialdesvinculación de la perspectiva universalista y, por otro, la tendencia más general a reducir los horizontes críticos de la perspectiva historiográfica a través de su homologación con la función tradicional de memoria de la historia y de la postulación de una trascendencia externa al registro propio de la disciplina. Al mantener la intención de reconstruir la historia de las clases subalternas, y al asumir los desafíos teóricos planteados por las nuevas corrientes - fundamentalmente la cuestión de los aspectos narrativos de la historiografía-, algunos desarrollos centrales de su obra permiten dar cuenta de una nueva inflexión en el registro de la vinculación entre reflexión sobre la memoria y autorreflexión de la práctica historiográfica.

En su principal obra, El queso y los gusanos, Ginzburg rescata la validez de las preocupaciones de una 'historia desde abajo', tratando de demostrar la 
posibilidad de realizar un estudio histórico de la cultura popular pese a las dificultades metodológicas de las fuentes: así, más allá de los problemas conceptuales y metodológicos inherentes a la definición tanto de qué constituye este abajo como de cuál es su relación con el arriba, y la correlativa preocupación por develar los márgenes de determinación y autonomía de las clases subalternas, esta perspectiva atisba a dar cuenta de un "clasismo genérico" divergente del indeterminismo del concepto de mentalidad, tanto en la versión del "utillaje mental" propuesto por Lucien Febvre como en su vuelco posterior al universo analítico de las representaciones sociales. Frente a las insuficiencias que encuentra en estas perspectivas analíticas, la elección de un caso "excepcional" se justifica por su "representatividad": el caso de Menocchio, un molinero sometido a juicio por la Inquisición, no es solamente excepcional por sus ideas y por la forma en que intenta difundirlas, sino también por la forma azarosa en que han llegado los testimonios acerca de él hasta nosotros. La existencia de este testimonio, y la imposibilidad del autor de establecer una filiación aceptable con las ideas del anabaptismo y el luteranismo constituyen las bases de la formulación de la pregunta por la existencia de una tradición popular de carácter oscuro para nuestro presente - "una corriente autónoma de radicalismo campesino que la agitación de la Reforma contribuyó a revelar, pero que era más antigua que la Reforma" - y, por derivación, para sostener la potencial representatividad del caso estudiado con respecto a muchos otros que permanecen inaccesibles ${ }^{25}$.

La historia de Menocchio se inscribe, en este sentido, en una incursión que transita un camino convergente con los aspectos exploratorios en los planos epistemológico y ético político del conocimiento histórico hallados en la obra de Thompson. Menocchio, al igual que los luditas o los defensores de una economía «moral» que Thompson rescataba desde la perspectiva de la «experiencia», es conceptuado como parte de una tradición sólo fragmentariamente inteligible, pero portadora de significados para la conciencia histórica del presente. Procurando dar cuenta de los componentes potencialmente emancipatorios de la 'tradición de los oprimidos', mediante un "juicio a posteriori" conscientemente reconocido, es "construido" por Ginzburg como un precursor que anticipa una serie de temas "que se convertirían en patrimonio de la cultura "progresista» del siglo siguiente: la aspiración a una renovación radical de la sociedad, la corrosión interna de la religión, la tolerancia”. Su restitución al objeto de la historia responde a la vez a un problema epistemológico y a un imperativo ético-político: no sólo testimonia la existencia de aspectos ininteligibles de las culturas del pasado, sino que da cuenta de una racionalidad alternativa que debe ser recuperada en vistas a dar cuenta de "una mutilación histórica de la que, en cierto sentido, nosotros mismos somos víctimas". De este modo, la intención restitutiva del historiador se ins- 
cribe en la línea del postulado benjaminiano que sostiene que "«nada de lo que se verifica se pierde para la historia», [...] mas «sólo la humanidad redenta toca plenamente su pasado»" ${ }^{26}$.

Al colocar los aspectos ético-políticos en el centro de la discusión epistemológica de la disciplina, el planteamiento de Ginzburg profundiza los rasgos autocríticos de la perspectiva historiográfica. Ya no se trata sólo del cuestionamiento a una idea de objetividad a-valorativa mediante el reconocimiento de los aspectos proyectivos de la propia experiencia como en el caso de Thompson, sino de un planteamiento que amplía este postulado al punto de asumir que todas las instancias del proceso de investigación son construidas: "la identificación del objeto y la de su relevancia; la elaboración de las categorías a través de las cuales éste se analiza; los criterios de prueba; los módulos estilísticos y narrativos a través de los cuales los resultados se transmiten al lector" ${ }^{27}$.

Este reconocimiento constituye el nudo del cuestionamiento a los aspectos insuficientes y distorsionados del cuadro general del pasado resultante de los modelos de historia total consolidados en la segunda generación de Annales sobre la base de la exploración cuantitativa y los casos típicos. Más allá de su confluencia epocalcon las tendencias al "desmigajamiento" de la historia y los retornos de diversos tópicos en general concebidos como antitéticos al modelo de totalidad - como el acontecimiento y la narración — la obra de Ginzburg no puede ser comprendida en el marco de estas tendencias: el rechazo a la totalidad se concibe más bien desde una postura radical que, problematizando la intervención del historiador en todas las instancias de la construcción del conocimiento, no reniega del objetivo universalista que fundamenta su práctica y, de este modo, desnuda los problemas prácticos que plantea a la conciencia del presente la asunción de un modelo en sí mismo insuficiente por razones estrictamente históricas, de acuerdo con su recepción de las tesis de Benjamin.

En este sentido, Ginzburg advierte explícitamente sobre algunas de las derivaciones posibles de este modelo como resultado de su ampliación al universo de las representaciones sociales - la exploración del 'tercer nivel' en términos de los principales exponentes de la tercera generación de la corriente-, reafirmando junto con el reconocimiento del papel del historiador en el proceso de conocimiento y los componentes narrativos de la historia, el papel normativo de las nociones de verdad y prueba. En El juez y el historiador, un texto escrito en un registro distinto y claramente orientado a una intervención en el presente, el autor señala la necesidad de reconsiderar la alternativa de la «comprensión» frente al «juicio» postulada y consensuada a partir de Bloch, habida cuenta los efectos que produjo con respecto al abandono de estas nociones inherentes a la historia en formulaciones teóricas y desarrollos historiográficos cada vez más orientados al análisis de "representaciones" y al 
estudio de las fuentes como testimonios de sí mismas sin vinculación constatable con el objeto representado. De este modo, sostiene una posición crítica frente al relativismo moral y el escepticismo cognoscitivo tendencial de las corrientes contemporáneas orientadas a concebir la realidad como construcción discursiva y a reducir el saber histórico a sus aspectos retóricos ${ }^{28}$.

Esta posición asume la perspectiva de la práctica historiográfica como espacio de construcción teórica y encuentra en la propia investigación histórica elementos que permiten establecer una filiación de los aspectos reductivos de las tendencias actuales con una particular recepción de la obra de Aristóteles a través de Nietszche ${ }^{29}$. Por otra parte, incluso la noción misma de "perspectiva» - que en la radicalización del giro antipositivista hacia posiciones antirrealistas actúa como un comodín de las tendencias potencialmente relativistas - es puesta en debate a partir de una indagación histórica, en un sentido que produce una nueva inflexión en la reflexión sobre la memoria: retomando los aspectos divergentes entre memoria e historia introducidos por Yosef Yerushalmi sobre la base de la constatación de la presencia fuerte de la memoria y la ausencia de una historiografía en la tradición judía, el examen de tres paradigmas básicos a partir de los cuales se pensó históricamente la cuestión de la perspectiva - los de la adecuación, el conflicto y la pluralidad - conduce en uno de sus últimos trabajos a una autorreflexión sobre la práctica historiográfica en la que el autor constata nuevamente los límites de la historia para dar cuenta de la experiencia que es capaz de evocar la memoria ${ }^{30}$.

Para Ginzburg, la memoria establece un nexo directo con la res gestae y es irreductible a la historia. De este modo, la postulación de la irreductibilidad de la memoria reafirma la necesidad de reconocer los límites del propio conocimiento para dar cuenta de la experiencia vivida, en la línea de las críticas a los aspectos distorsionadores de la resolución falaz del problema de la construcción de una interpretación de la totalidad. Sin embargo, en aquel caso, el reconocimiento de las limitaciones del presente para dar cuenta del pasado se anudaba con el imperativo ético de una restitución que, aunque fragmentaria, resultaba posible. De este modo, el planteamiento sobre la irreductibilidad de la memoria a la historia desde el propio registro de la investigación histórica puede leerse en el sentido de que éste es el camino para postular la existencia de aquellos aspectos irreductibles que la reflexión sobre la distancia que separa al presente del pasado, es decir, la historia, no puede representar. La historia se presenta entonces explícitamente como una perspectiva autoconsciente de sus límites: si frente a la experiencia del pasado, a veces la memoria dice más que la historia, frente a otras perspectivas que buscan dar cuenta del fenómeno mismo de la memoria, la historia se revela como una perspectiva que, desde el reconocimiento de la irreductibilidad de 
Hernán Sorgentini

aquella experiencia en torno a la cual constituye su objeto, presenta sólidas credenciales teóricas y epistemológicas para su efectivo abordaje.

\section{IV}

El debate actual en torno al problema de la memoria presenta una amplia gama de estudios que han tendido a desarrollarse profusamente en los últimos años como consecuencia de varios factores. Entre estos factores, cabe mencionar en un lugar destacado para los países de América Latina el desafío que presentan al conocimiento y a la acción de una memoria voluntaria y militante las dificultades de interiorización y significación de experiencias de carácter límite originadas en las prácticas del terrorismo de Estado. De este modo, en la discusión de estos problemas, a las dificultades epistemológicas del conocimiento histórico para resolver el problema de los aspectos oscuros o sólo fragmentariamente inteligibles del pasado parecen sumarse, en este caso, las de la urgencia práctica de la constitución de una significación de acontecimientos de complejas pervivencias en el presente. En esta significación pueden intervenir tanto la memoria como la historia. Si a veces la memoria, por el tipo de vinculación directa que establece con la experiencia vivida resulta un instrumento adecuado para esclarecer algunos de estos aspectos, en otras oportunidades, por desprenderse de la pretensión de verdad que guía a la historia, oblitera otros, como puede ser el caso del escaso debate sobre la radicalización política de los años 70 en la memoria instituida en los primeros años de la transición democrática en la Argentina y el consecuente despojamiento de la subjetividad política de las víctimas del terrorismo de Estado.

La revisión de los núcleos centrales de la renovación historiográfica considerados da cuenta de un espacio de articulación entre estas perspectivas y, en este sentido, puede contribuir a iluminar tanto sus registros divergentes como sus posibilidades de diálogo. En Bloch, el examen crítico de la memoria como objeto se presenta como un camino que orienta la redefinición de la historia y su función práctica en el contexto de una crisis de legitimidad de la disciplina. Desechando la función práctica implícita en el objetivismo ingenuo de la historiografía tradicional, la anteposición del problema de la comprensión a cualquier posibilidad de juicio articula la función de memoria de la historia en un segundo momento del análisis: un momento que requiere agotar previamente el problema de las funciones de saber de la disciplina y que sólo se concibe diferidamente en relación con el contenido universalista de la tradición nacional. La incorporación del problema de la subjetividad aportado por la perspectiva thompsoniana profundiza los horizontes de esta dimensión práctica al tiempo que redefine la oposición entre juicio y com- 
prensión a partir de una consideración de los aspectos ético-políticos intrínsecos a la reconstrucción histórica. A partir de este planteamiento, para comprender es necesario recurrir al juicio. El juicio reconoce los aspectos proyectivos de la propia experiencia del historiador, pero se justifica en última instancia en su capacidad epistémica para acercarnos al objetivo siempre diferido de, para decirlo en los términos tradicionales, "contar los hechos tal cual ocurrieron". De este modo, la opción thompsoniana fundamenta una articulación de la memoria no ya sólo como función práctica del saber histórico o de una genérica apelación al contenido universalista de la tradición nacional, sino también de un sujeto potencialmente emancipador que se reconoce en ella y a la vez la asume críticamente al rechazar simétricamente las falacias de una identificación transhistórica con su racionalidad intrínseca y los aspectos clausurantes implícitos en la pseudo-objetividad de una supuesta razón histórica. En Ginzburg, esta redefinición parece dar un nuevo giro al incorporar el reconocimiento radical de la construcción del historiador en todas las etapas del proceso de conocimiento y, con ello, una reflexión sobre los límites del conocimiento histórico para dar cuenta de la vinculación directa entre memoria y experiencia: de este modo, la divergencia inherente a los registros de la historia y la memoria permite vislumbrar en este último un poder iluminador que alerta sobre los aspectos irrestituibles de la experiencia en el registro del conocimiento sistemático y funciona, por tanto, como un potencial contrapunto crítico para señalar los límites de su capacidad comprensiva y de sus posibilidades de constituir una guía para la práctica.

Más allá de la distintas inflexiones que denota la incorporación de estos elementos a la autorreflexión sobre la disciplina, en todos los casos se mantiene una articulación con la función normativa de la noción de verdad. El mantenimiento de la verdad como intención siempre diferida y, a la vez, como principio normativo, permite desnudar tanto las falacias de la orientación práctica implícita en la (pseudo) comprensión a-valorativa del oficio del historiador legitimado exclusivamente en la observancia de una serie de métodos y procedimientos de carácter formal, como de los problemas de una orientación práctica de la memoria voluntaria sin validación en el saber histórico. En este sentido, contiene un potencial teórico capaz de situar en un horizonte crítico común el reconocimiento de los límites del propio conocimiento histórico y los aspectos falaces de la memoria, sobre la base de un mejor planteamiento de las preguntas sobre las perpetuas irresoluciones y los imperativos ético-políticos siempre renovados de dos empresas que por divergentes no son necesariamente antitéticas: la de significar la experiencia y la de comprender como sucedieron efectivamente las cosas. 
Hernán Sorgentini

\section{NOTAS}

'En estas críticas confluyen los señalamientos sobre las insuficiencias explicativas de la historia frente a las ciencias sociales y la denuncia del carácter pernicioso de la función práctica asociada a sus versiones tradicionales. Frente a ellas, la obra de Bloch articula una apertura a un diálogo con las ciencias sociales y el mantenimiento de un registro propio de la disciplina. Análisis específicos sobre la escuela de Annales y la obra de Bloch pueden hallarse en DOSSE, Françoise. La historia en migajas. De «Annales» a la «nueva historia», Valencia: Edicions Alfons el Magnànim, 1988; BURKE, Peter. La revolución historiográfica francesa. La Escuela de los Annales: 1929-1989, Barcelona: Gedisa, 1993; AGUIRRE ROJAS, Carlos Antonio. La Escuela de los Annales. Ayer, Hoy, Mañana, España: Montesinos, 1999; GEREMEK, Bronislaw. Marc Bloch, historiador y resistente, Buenos Aires: Biblos, 1990. Sobre las formas particulares en que Bloch construye su defensa de la disciplina histórica puede verse el Prefacio de Jacques Le Goff a la última edición de Apología para la historia. LE GOFF, J., "Prefacio". En BLOCH, Marc. Apología para la historia o el oficio de historiador [Edición crítica preparada por Étienne BLOCH], México: Fondo de Cultura Económica, 1996, pp. 43-76. Sobre las funciones de saber y memoria de la historia en su obra y el problema de la transmisión de los recuerdos pueden hallarse análisis más exhaustivos en NOIRIEL, Gérard. Sobre la crisis de la historia, Madrid: Cátedra, 1997 y, sobre todo, en MASTROGREGORI, Massimo. El manuscrito interrumpido de Marc Bloch. Apología para la historia o el oficio de historiador, México: Fondo de Cultura Económica, 1998.

${ }^{2}$ HALBWACHS, Maurice. Les Cadres Sociaux de la Mémoire, Paris: Libraire Félix Alcan, 1925. La obra que toma Halbwachs como punto fundamental de referencia es BERGSON, Henri. Matière et mémoire. Essai sur la relation du corps à l'esprit, Paris: Félix Alcan, 1896 [Trad. cast. Materia y Memoria. Ensayo sobre la relación del cuerpo con el espíritu, Madrid: Librería de Victoriano Suárez, 1900]. La orientación de la reflexión de Halbwachs hacia el estudio de los distintos grupos tal vez explique las razones — por otra parte justas — por las cuales es hoy considerado un referente clásico central de la sociología de la memoria. Sin embargo, una lectura más detenida de su obra, permite dar cuenta del sentido filosófico que entraña su reflexión sobre la memoria en relación con una teoría del "pensamiento social". Hemos desarrollado más exhaustivamente una interpretación sobre la "reescritura" que Halbwachs hace de la obra de Henri Bergson en PÉREZ, Alberto y SORGENTINI, Hernán. "Perspectivas para una exploración de la conexión memoria-historia", trabajo presentado en las VIII ${ }^{\circ}$ Jornadas Interescuelas Departamentos de Historia, Universidad Nacional de Salta, Salta, 19-22 de Septiembre de 2001, inédito. Una referencia a la centralidad de la obra de Bergson en relación con la cuestión de la memoria y la obra clásica de Halbwachs puede hallarse en RICOER, Paul. "L'écriture de l'histoire et la représentation du passé”. Annales. Histoire, Sciences Sociales, 55e Année, nº 4, pp. 731-747, Juillet-Août 2000 y MARCEL, J. C. y MUCHINELLI, L. "Un fondement du lien social: la mémoire collective selon Maurice Halbwachs". Technologies. Idéologies. Pratiques. Revue d'anthropologie des connaissances, 13 (2), pp. 63-88, 1999.

${ }^{3} \mathrm{BLOCH}$, Marc. "Memoria colectiva, tradición y costumbre. A propósito de un libro re- 
ciente". En BLOCH, Marc. Historia e historiadores, [textos reunidos por Ètienne BLOCH], Madrid: Akal, 1999, pp. 223-232 [publicado originalmente en Revue de synthèse, t. XL, pp. 73-83, diciembre de 1925]. Con respecto al problema de la continuidad de las tradiciones, Bloch señala la limitada base historiográfica de las conclusiones de Halbwachs y observa las innovaciones que se esconden "bajo el nombre de costumbres inmemoriales", así como los cambios en tradiciones como el cristianismo que "se transformó mucho más profundamente, entre la Paz de la Iglesia y la Reforma, de lo que Halbwachs parece imaginar". Idem., pp. 227-231.

${ }^{4}$ HALBWACHS, Maurice. La mémoire collective, Paris: Presses Universitaires de France, 1950, especialmente pp. 78 y ss. Sobre la concepción tradicional de la historia de Halbwachs puede verse BURKE, Peter. "La historia como memoria colectiva". En BURKE, Peter, Formas de historia cultural, Madrid: Alianza, 2000, pp. 65-85, esp. p. 67.

${ }^{5} \mathrm{Cfr}$. "Reflexiones de un historiador acerca de los bulos surgidos durante la guerra" y "La vida de ultratumba del rey Salomón”, reunidos también en BLOCH, Marc. Historia e historiadores, op. cit., pp. 175-197 y 198-222. Sobre estos ensayos, que Bloch pensaba reunir en un volumen sobre la tarea de los historiadores destinado a un público más vasto que el conformado por los especialistas de la disciplina y que propuso sin éxito al editor Gallimard en 1932, cfr. MASTROGREGORI, Massimo. Op.cit., cap. 2

${ }^{6} \mathrm{BLOCH}, \mathrm{Marc}, \mathrm{La}$ sociedad feudal. La formación de los vínculos de dependencia, México: UTEHA, 1958, p. 108.

${ }^{7}$ Un análisis exhaustivo sobre estos problemas, y específicamente sobre las distintas redacciones del manuscrito y sobre el contexto específico en que fue publicado por Lucien Febvre en 1949, puede hallarse en MASTROGREGORI, Massimo. Op. cit. Para un análisis de la cuestión de la función de memoria de la historia pensada como "acto de comunicación" en Bloch cfr. NOIRIEL, Gérard. Op. cit., pp. 83-91.

${ }^{8}$ BLOCH, Marc. Apología..., op. cit.., pp. 147-148

${ }^{9}$ Cfr. LE GOFF, Jacques. Op. cit, esp. pp. 55 y 62 y BLOCH, Marc. Apología..., op. cit., pp. 140 y 247 y ss.

${ }^{10} \mathrm{BLOCH}$, Marc. L'étrange défaite. Témoignage écrit en 1940, Paris: Gallimard, 1990, esp. p. 31. Cfr. también el Prefacio de Stanley Hoffman en pp. 11-26.

${ }^{11}$ Hemos tratado algunos de estos problemas en SORGENTINI, Hernán. "La recuperación de la experiencia histórica: un comentario sobre E. P. Thompson", Sociohistórica. Cuadernos del CISH, La Plata, n 7, pp. 53-80, Primer Semestre de 2000, y "Racionalidad de la acción, valoración y reconstrucción histórica en la historiografía de E. P. Thompson”, Storiografia. Rivista anuale di storia, Roma-Pisa: Instituti Editoriali e Poligrafici Internazionali, vol. V, 2001. Dentro de la vasta discusión originada en torno a estas cuestiones pueden citarse entre otros THOMPSON, Edward P. The Poverty of Theory: or an Orrery of Errors, London: Merlín Press, 1978 [Miseria de la teoría, Barcelona: Crítica, 1981]; ANDERSON, Perry. Arguments within English Marxism, Londres: Verso, 1980 [Teoría, política e historia. Un debate con E. P. Thompson, Madrid: Siglo XXI, 1986]; JOHNSON, Richard. "Edward 
Thompson, Eugene Genovese y la historia socialista-humanista”. En JOHNSON, R. et. al. Hacia una historia socialista [compilación de Rafael Aracil y Mario García Bonafé], Barcelona: Ediciones del Serbal, 1983, pp. 52-85; MCCLELLAND, Keith. “Algunos comentarios sobre 'Edward Thompson, Eugene Genovese y la historia socialista-humanista”. En JOHNSON, Richard, et al, op. cit., pp. 87-108; WOOD, Ellen Meiksins. "El concepto de clase en E. P. Thompson”, Cuadernos Políticos, México D. F., nº 36, pp. 87-105, abril-junio de 1983 y "Falling Through the Craks: E. P. Thompson and the Debate on Base and Superestructure”. En KAYE, Harvey y MCCLELLAND, Keith (eds.). E. P. Thompson. Critical Perspectives. Filadelfia: Temple, 1990, pp. 125-152; SEWELL JR., William H., "How Classes are Made: Critical Reflections on E. P. Thompson's Theory of Working-Class Formation”. En KAYE, Harvey y MCCLELLAND, Keith. Op. cit., pp. 50-77; SAZBÓN, José. “Dos caras del marxismo inglés: el intercambio Thompson-Anderson”, Punto de vista, Buenos Aires, año X, $\mathrm{n}^{\circ}$ 29, pp. 11-26, abril-julio de 1987; CAÍNZOS LÓPEZ, Miguel A. "Clase, acción y estructura: de E. P. Thompson al posmarxismo”, Zona Abierta, Madrid, n 50, pp. 1-69, eneromarzo de 1990; KAYE, Harvey. "E. P. Thompson, the British Marxist Historical Tradition and the Contemporary Crisis". En The Education of Desire. Marxists and the Writing of History, New York-London: Routledge, pp. 98-115; BENÍTEZ MARTíN, Pedro. E. P. Thompson y la historia. Un compromiso ético y político, Madrid: Talasa, 1996.

${ }^{12}$ Sobre este punto resultan ilustrativos el trabajo de HOBSBAWM, Eric. "El Grupo de Historiadores del partido comunista”, Historia Social, Valencia, n 25, pp. 61-81, 1996, y los estudios de KAYE, Harvey. The British Marxist Historians. An Introductory Analysis, Cambridge: Polity Press, 1984. [Los historiadores marxistas británicos, Zaragoza: Prensas Universitarias de Zaragoza, 1984] y DWORKIN, Dennis, Cultural Marxism in Postwar Britain. History, the New Left and the Origins of Cultural Studies, Durham and London: Duke University Press, 1997.

${ }^{13}$ THOMPSON, Edward Palmer. The Making of the English Working Class, New York: Vintage, 1966 [1963] [La formación de la clase obrera en Inglaterra, 2 Tomos, Barcelona: Crítica, 1989]. En adelante se cita la edición castellana. Para el tratamiento dado al jacobinismo inglés cfr. la Primera Parte, especialmente los capítulos 1 y 5; la vinculación de esta ruptura en la subjetividad con el problema de la determinación estructural se traza fundamentalmente en el capítulo 6; la tesis del papel validatorio de la interpretación thompsoniana que tiene la recuperación de la «experiencia de clase» frente a la (pseudo)objetividad de las interpretaciones liberales se desprende del capítulo final de la Segunda Parte, especialmente pp. 494-495.

${ }^{14}$ Idem., Tomo II, p. 127.

${ }^{15}$ Idem., Tomo II, pp. 59-60.

${ }^{16}$ THOMPSON, Edward P. "Eighteenth-century English society: Class struggle without class?”, Social History, Vol III, n 2, pp. 133-165, mayo de 1978. [Trad. cast. "La sociedad inglesa del siglo XVIII: ¿lucha de clases sin clases?”, en THOMPSON, E. P. Tradición, revuelta y consciencia de clase. Estudios sobre la crisis de la sociedad preindustrial, Barcelona: Crítica, 1979, pp. 13-61]. 
${ }^{17}$ THOMPSON, Edward. P. Costumbres en común, Barcelona: Crítica, 1995, citas en pp. 13 y 18-19. Una interesante lectura de esta obra puede hallarse en WOOD, Ellen Meiksins. “Custom Against Capitalism”, New Left Review, n 195, sep-oct. 1992, pp. 21-28.

${ }^{18}$ Cfr. THOMPSON, Edward P. Costumbres..., op. cit., p. 588. El rastreo de esta antigua polémica con Williams, desarrollada en su reseña de The Country and the City de 1961, constituye un punto clave no sólo para situar las divergencias teóricas entre las dos grandes vertientes a partir de las cuales se configura la nueva izquierda británica con posterioridad a 1956 y sus derivaciones en los caminos de la historiografía socialista y el culturalismo, sino para señalar cómo la defensa de la perspectiva propia de la historia y la adscripción al marxismo constituyen la singularidad de la interpretación thompsoniana y explican sus múltiples flancos polémicos. En este sentido, puede afirmarse que es sobre este particular concepto de cultura como "whole way of struggle" que se concibe una "conciencia de la costumbre", en el registro de una tradición que puede verse ahora como espacio en el que se desarrolla la lucha de clases y como objeto de disputa. Cfr. THOMPSON, Edward P. "Country and City". En THOMPSON, Edward P. Making History. Writings on History and Culture, New York: The New Press, 1994, pp. 242-253. Un tratamiento teórico similar tiene en otra obra dedicada al siglo XVIII el principio del "dominio de la ley", cuya potencial orientación transformadora se sostiene - frente a las críticas estructuralistas que absolutizan el carácter mistificador de su formalismo - a partir de la iluminación retrospectiva provista por la constatación de la ruptura marcada por su abandono por parte de las clases dominantes, ruptura situada, también aquí, en la década de 1790. Cfr. THOMPSON, Edward P. Whigs and Hunters. The Origin of the Blck Act, New York: Pantheon Books, 1975.

${ }^{19}$ Sobre este punto, la principal obra de referencia es THOMPSON, Edward P. Miseria..., op. cit. Cfr. También THOMPSON, E. P. “An Open Letter to Leszek Kolakowsky”, Socialist Register, Londres, 1973.

${ }^{20} \mathrm{Un}$ balance sobre este punto referido al ámbito anglosajón puede hallarse en DWORKIN, D. Op. cit., esp. cap. 5 "History from below".

${ }^{21}$ Un balance sobre las virtudes y dilemas que plantea esta apertura del objeto de la historia desde abajo puede hallarse en SHARPE, Jim. "Historia desde abajo". En BURKE, Peter (comp.). Formas de hacer historia, Madrid: Alianza, 1993. Eric Hobsbawm ha definido la perspectiva marxista, señalando la eficacia de las anteojeras con las que los autores inscriptos en esta tradición recortan este problema en torno a la pregunta articuladora por el sujeto. Cfr. HOBSBAWM, Eric. "History from Below - Some Reflections", en KRANTZ, Frederick (ed.). History from Below. Studies in Popular Protest and Popular Ideology, Oxford and New York: Basil Blackwell, 1988, pp. 13-27. Vinculado con esta defensa de la perspectiva marxista puede leerse también su rescate del universalismo de la historia en HOBSBAWM, Eric. “Identity History Is Not Enough", en HOBSBAWM, Eric. On History, New York: The New Press, 1997, 266-277. [Publicado previamente con el título "The Historian between the Quest for the Universal and the Quest for Identity”, Diogenes, 42/2, 1994].

${ }^{22} \mathrm{Un}$ rastreo de estas tendencias puede hallarse en DWORKIN, D. Op. cit. Los planteamientos que permanecen en adhesión al marxismo tienden, en general, a fundamentar los as- 
pectos teóricos de su producción en un registro externo al de las implicancias teóricas de la historiografía, fundamentalmente valorizando el diálogo con el pensamiento marxista estructuralista. Cfr. por ejemplo JOHNSON, Richard. Op. cit.. Una sugerente interpretación sobre la filiación entre las dos tendencias puede hallarse en WOOD, E. M., "Falling...", op. cit.

${ }^{23}$ SAMUEL, Raphael. Theatres of Memory, Volume 1: Past and Present in Contemporary Culture, London-New York: Verso, 1994, p. X.

${ }^{24}$ NORA, Pierre (dir.), Les lieux de mémoire, 7 vols., Paris: Gallimard, 1984-1992, cfr. especialmente el prefacio de Nora: "Entre Mémoire et Histoire. La problématique des lieux", Tomo I: “La République”, p. XXIII.

${ }^{25}$ GINZBURG, Carlo. El queso y los gusanos. El cosmos, según un molinero del siglo XVI, Barcelona: Muchnik, 1994, esp. "Prefacio", pp. 9-24 y pp. 52-53.

${ }^{26}$ Idem, pp. 23-24. La cita de Benjamin corresponde a las “Tesis de filosofía de la historia”. Cfr. BENJAMIN, Walter. La dialéctica en suspenso. Fragmentos sobre la historia, Santiago de Chile: Universidad ARCIS y LOM, 1995. Un análisis de la obra de Ginzburg que informa muchos de los puntos planteados en este apartado puede hallarse en el estudio de SERNA, Justo y PONS, Anaclet. Como se escribe la microhistoria. Ensayo sobre Carlo Ginzburg, Madrid: Cátedra, 2000.

${ }^{27}$ GINZBURG, Carlo. "Microhistoria: dos o tres cosas que sé de ella”, Entrepasados. Revista de Historia, Buenos Aires, Año V, nº 8, pp. 51-73, comienzos de 1995, cita en p. 67.

${ }^{28}$ GINZBURG, Carlo. El juez y el historiador. Consideraciones al margen del proceso Sofri, Madrid: Anaya y Mario Muchnik, 1993, esp. pp. 18-24. Cfr. también su intervención en la discusión sobre el problema de la representación del pasado y el problema de la Shoa en GINZBURG, Carlo. “Just One Witness”. En FRIEDLANDER, Saul (ed.), Probing the Limits of Representation, Cambridge, Mass.: Harvard University Press, 1992, pp. 82-96

${ }^{29}$ GINZBURG, Carlo. History, Rhetoric, and Proof. The Menahem Stern Jerusalem Lectures, Hanover and London: University Press of New England, 1999. Ver especialmente la introducción y el cap. 1 "Aristotle and History, Once More".

${ }^{30}$ GINZBURG, Carlo. “Distance and perspective: Two Metaphors”. En GINZBURG, Carlo. Wooden Eyes. Nine reflections on Distance, New York: Columbia University Press, 2001. [Trad. cast. "Distancia y perspectiva. Dos metáforas", Entrepasados. Revista de Historia, Año VIII, n 16, pp. 99-121, princ. de 1999]. Este aspecto irreductible de la memoria es subrayado también en un breve artículo sobre Halbwachs. GINZBURG, Carlo. "Shared Memories, Private Recollections", History and Memory. Studies in Representations of the Past, Indiana: Indiana University Press, Vol. 9, nº 1/2, pp. 353-363, 1997.

Artigo recebido em 2/2003. Aprovado em 4/2003. 